Research Paper

\title{
Eukaryotic translation initiation factor 5A-2 involves in doxorubicin-induced epithelial-mesenchymal transition in oral squamous cell carcinoma cells
}

\author{
Liang Fang ${ }^{\bowtie}$, Li Gao, Lei Xie, Guizhou Xiao \\ Department of head and neck surgery, Sir Run Run Shaw Hospital, School of Medicine, Zhejiang University, 3 East Qingchun Road, Hangzhou 310016, P.R. \\ China \\ $\triangle$ Corresponding author: Liang Fang MD., Ph.D., Department of head and neck surgery, Sir Run Run Shaw Hospital, School of Medicine, Zhejiang University, 3 \\ East Qingchun Road, Hangzhou, 310016, P.R. China. Tel: +86 (0)571 86002186; Fax: +86 (0)571 86002186; E-mail: fangliang_dr@zju.edu.cn. ORCID: \\ 0000-0003-1473-146476 \\ (C) Ivyspring International Publisher. This is an open access article distributed under the terms of the Creative Commons Attribution (CC BY-NC) license \\ (https://creativecommons.org/licenses/by-nc/4.0/). See http://ivyspring.com/terms for full terms and conditions.
}

Received: 2018.03.18; Accepted: 2018.07.18; Published: 2018.09.08

\begin{abstract}
Background: Epithelial-mesenchymal transition (EMT) is considered to be vital during chemotherapy resistance in oral squamous cell carcinoma (OSCC). Recently, eukaryotic initiation factor 5A-2 (elF5A-2), a potential oncogene, has been reported to be involved in chemotherapy resistance in human cancers.

Materials and Methods: N1-guanyl-1,7-diaminoheptane (GC7, a novel elF5A-2 inhibitor) or siRNA on responses to doxorubicin were examined in OSCC cells. Cytotoxicity and protein expression were evaluated by CCK-8 and EdU incorporation assay and western blotting. Tca8113 cells were used for establishment and treatment of tumor xenografts in vivo.

Results: Low concentration of GC7 $(5 \mu \mathrm{M})$ significantly enhanced doxorubicin cytotoxicity in both epithelial phenotype OSCC cells (Cal27) and mesenchymal phenotype OSCC cells (HN30 and Tca8113). EMT process promoted by doxorubicin in Cal27 cells could be reversed by GC7. Additionally, GC7 induced mesenchymal-epithelial transition (MET) in HN30 and Tca8113 cells. Silencing of elF5A-2 by specific siRNA exhibited the similar effects. The synergistic cytotoxicity of doxorubicin/GC7 combination was not induced in Twist-1, an EMT driving factor, silenced Cal27, HN30, and Tca8113 cells. GC7 also synergized doxorubicin to inhibit tumor growth in vivo treatment.

Conclusions: Our study strongly proved that combined treatment with GC7 may boost the therapeutic effect of doxorubicin in OSCC by inhibiting the EMT.
\end{abstract}

Key words: Eukaryotic initiation factor 5A-2 (eIF5A-2), N1-guanyl-1,7-diaminoheptane (GC7), Oral squamous cell carcinoma (OSCC), Epithelial-mesenchymal transition (EMT), doxorubicin, chemo-resistance

\section{Background}

Oral cancer is a highly malignant tumor type and is the main threat to human health and quality of life worldwide with oral squamous cell carcinoma (OSCC) accounting for the majority of oral cancer diagnoses $[1,2]$. It has been shown that surgery is the optimal therapeutics for early OSCC and is often used in combination with chemotherapy or radiotherapy in late cases to prevent recurrence and to improve treatment result. However, despite numerous promising chemotherapy regiments and molecular-targeted based therapies, the prognosis of oral cancer remains poor due to increased chemo-resistance capacity to traditional chemotherapy agents and adverse side-effects. The traditional chemotherapy agent doxorubicin is commonly used in the treatment of OSCC and other malignant tumors. Recently, several reports have demonstrated that the combined treatment of doxorubicin and novel molecular-targeted agents enhance the chemotherapeutic effect [3-7].

Tumorigenesis in OSCC is a multistep progression and exhibits various morphological and 
molecular features which are considered to be controlled by some abnormally expressed genes. Loss of epithelial characteristic like barrier functions conducted by cell-cell junctions and gain of mesenchymal characteristic including metastatic ability is one such change, which indicates initiation of epithelial-mesenchymal transition (EMT). In the process of EMT, many epithelial markers are down-regulated, such as E-cadherin, beta-catenin, claudins, desmoplakin, occluding, and cytokeratins, meanwhile various mesenchymal markers are up-regulated, such as Vimentin, N-cadherin, Snail-1/2, and Fibronectin. The EMT, inducing epithelial phenotype cells to transform to the mesenchymal phenotype, is a complex and reversible process which has been recognized as a response for the acquisition of metastasis and chemo-resistance in oral cancer [8]. Emerging evidence suggests that overexpression of mesenchymal-related genes in OSCC, including ZEB1, ZEB2, Snail, and Twist, is also related to poor survival $[9,10]$. These studies reveal that EMT may play a vital role in the non-ideal effect of chemotherapeutic agents in OSCC. Other reports have detected that the EMT progress in various human malignant tumors could be induced by doxorubicin, which may contribute to chemo-resistance to subsequent chemotherapy [11-14]. Therefore, as the oncogenic potential of EMT, it is important to explore whether OSCC cells undergo the EMT process after doxorubicin treatment.

Eukaryotic translation initiation factor 5A-2 (eIF5A-2), has been shown to participate in the translation of several proteins which are associated with cell proliferation, cancer progression, invasiveness, and metastasis, thus is considered to be a novel oncogene in various human cancers [15-20]. Deoxyhypusine synthase (DHPS), a hypusination catalyzing enzyme which was revealed by tumor metastasis-related genes analysis, contributes to the development of high malignancy and poor prognosis. At present, eIF5A-2 is the main substrate of DHPS and inhibiting eIF5A-2 might be an effective strategy for the enhancement of the effect of current anti-cancer agents. Moreover, eIF5A-2 was identified to involve in EMT progression in several human cancers, such as colorectal carcinoma [12] and HCC [21]. Thus, it is of great importance to investigate the potential correlation of EMT with eIF5A-2 in OSCC.

Recently, N1-guanyl-1,7-diaminoheptane (GC7), an agent inhibiting DHPS activity, exerts significant suppression of proliferation by inhibiting eIF5A-2 in several human cancers[13, 22-24]. In the present research, we aimed to explore the chemotherapeutic effect of doxorubicin-based treatment plus GC7 in OSCC cells and found that GC7 increased doxorubicin chemosensitivity in OSCC cells. We also explored the potential molecular mechanisms when doxorubicin was co-administrated with GC7 and found that doxorubicin-induced EMT was significantly suppressed via GC7 mediated inactivation of eIF5A-2 in OSCC cells.

\section{Materials and Methods}

\section{Cell culture and reagents}

The human OSCC cell lines, Cal27, HN30 and Tca8113, were purchased from Chinese Oral Tissue Culture and Collection Center (Shanghai, P.R. China) which were sustained as monolayer in RPMI-1640 medium (Gibco, Carlsbad, CA) with $1 \%$ streptomycin/penicillin (Sigma; St. Louis, MO) and 10 $\%$ fetal bovine serum (FBS; HyClone, Logan, UT) in 5 $\% \mathrm{CO}_{2}$ at $37{ }^{\circ} \mathrm{C}$. Doxorubicin was obtained from Sigma (St. Louis, MO) and stock solutions were prepared in DMSO. GC7 were purchased from Calbiochem Merck Group (Darmstadt, Germany). Unless else indicated, all other reagents were purchased from Sigma of the purest grade available.

\section{CCK-8 assay and EdU incorporation assay}

OSCC cells or OSCC cells transfected with specific siRNA were cultured on 96-well plates $\left(3 \times 10^{3}\right.$ cells/well) and allowed to attach for $12 \mathrm{~h}$, serum starved overnight to synchronize the cells, then the culture media was changed to the whole medium including the Doxorubicin or Doxorubicin combined with GC7 at indicated doses for addition $48 \mathrm{~h}$. Next, cell counting kit-8 (10 $\mu \mathrm{L} /$ well, Dojindo, Kumamoto, Japan) was used following the protocol. Briefly, the cells with CCK-8 solution were incubated at $37^{\circ} \mathrm{C}$ for $3 \mathrm{~h}$, and absorbency was detected via the MRX II microplate reader (Dynex, Chantilly, VA) at $450 \mathrm{~nm}$. Cell viability was estimated as percentages of blank control cells. Click-iT EdU Imaging Kit (Invitrogen, Carlsbad, CA) was used to measure the inhibitive ratio of cell viability following the product protocol.

\section{siRNA Transfection}

eIF5A-2 siRNA, Twist siRNA or negative control siRNA (Santa Cruz, Dallas, TX) was transfected to OSCC cells via Lipofectamine 2000 (Invitrogen, Carlsbad, CA) following the manufacturer's protocol. The transfection culture medium (Opti-MEM, Gibco, Carlsbad, CA) was changed to whole medium $6 \mathrm{~h}$ after transfection, and the cells were incubated for an additional $24 \mathrm{~h}$ before all following experiments.

\section{Western blotting analyses}

OSCC cells were lysed in $50 \mu \mathrm{l}$ cell lysis buffer (Cell signaling, Danvers, MA) containing protease 
inhibitors (BioVision) according to the manufacturer's instructions. The cell lysates were quantified using the BCA Protein Kit (Thermo Fisher Scientific, Rockford, IL). $10 \%$ SDS-PAGE was used to separated cell lysates and proteins were transferred to PVDF membranes (Millipore, Billerica, MA), blocked with TBS/T containing $5 \% \mathrm{BSA}$ for $2 \mathrm{~h}$ on ice. The membranes were incubated with indicated primary antibodies (Vimentin, E-cadherin, eIF5A-2, Twist or GAPDH, dilution 1:1000, Abcam, Cambridge, MA) at $4{ }^{\circ} \mathrm{C}$ overnight. After washing three times with TBS/T, the membranes were incubated with the specific HRP-conjugated secondary antibodies (Abcam, Cambridge, MA, dilution 1:2000) at room temperature for $1 \mathrm{~h}$. The protein expression was detected by chemiluminescence (GE Healthcare, Piscataway, NJ) and imagined by autoradiography (Kodak, Rochester, NY). The quantifications of protein were done by estimation of protein bands densities (TIFF images) using Image-Pro Plus 6.0 software (Media Cybernetics, Bethesda, MD) and protein expression was standardized to GAPDH.

\section{Establishing and management of tumor xenografts in vivo}

All animal studies were proved by the ethics committee of Sir Run Run Shaw Hospital, School of Medicine, Zhejiang University and performed in compliance with the Guidelines for the Care and Use of Laboratory Animals of Zhejiang University, P.R. China. Male athymic BALB/c nude mice (Shanghai Experiment Animal Centre, PR China), 5-6 weeks old, 15-20 g weight, were used in the research. OSCC Tca8113 cells were used to establish tumor xenografts in vivo following the procedure previously described [25]. Drug interventions were initiated 2 weeks after OSCC cells injection. OSCC Tca8113 cells injected nude mice were randomly divided into four groups (5/group): three research groups including GC7 (1 $\mathrm{mg} / \mathrm{kg})$, Doxorubicin $(4 \mathrm{mg} / \mathrm{kg})$ and GC7 $(1 \mathrm{mg} / \mathrm{kg})$

+ Doxorubicin $(4 \mathrm{mg} / \mathrm{kg})$, together with one vehicle-treated negative control group. Drug interventions were provided by i.p. every 2 days for 2 weeks. Subsequently, tumor specimens were carefully dissected for tumor weights measurement after euthanization via cervical dislocation. The relative tumor inhibitory ratio was calculated by the following formula: (mean tumor weight of negative control group - mean tumor weight of each research group)/ (mean tumor weight of negative control group) $\times 100 \%$.

\section{Statistical analyses}

The data were presented as mean \pm standard deviation (SD). Results were statistically analyzed using SPSS 17.0 software (SPSS Inc., Chicago, IL). Bonferroni's post hoc test following two-way ANOVA was used to compare the effects of combined treatment. Student's t-tests were carried out for the statistic difference between the two groups. Statistical significance was considered acceptable at $\mathrm{p}<0.05$.

\section{Results}

\section{Low concentrations of GC7 had little effect on OSCC cell viability}

Inhibiting DHPS mediated eIF5A-2 hypusination, GC7 specifically prevents the activation of eIF5A-2. However, the cytotoxicity of GC7 against OSCC cells has not been reported. Cell counting kit-8 assays were carried out to investigate the cytotoxicity of GC7 in Tca8113, Cal27, and HN30 OSCC cell lines. Our results revealed that GC7 $(0-5 \mu \mathrm{M})$ had little effect on Cal27 and HN30 cell viability, however significantly inhibited cell viability at its concentration exceeding $10 \mu \mathrm{M}$ (Figure 1A, C). Similarly, cell viability of Tca8113 cells was well when its concentration was less than $10 \mu \mathrm{M}$, while significantly inhibited at the GC7 concentration exceeding $20 \mu \mathrm{M}$ (Figure 1B). Our data indicated that GC7 at low concentrations exerted almost no cytotoxic effect against OSCC cells. Several reports have
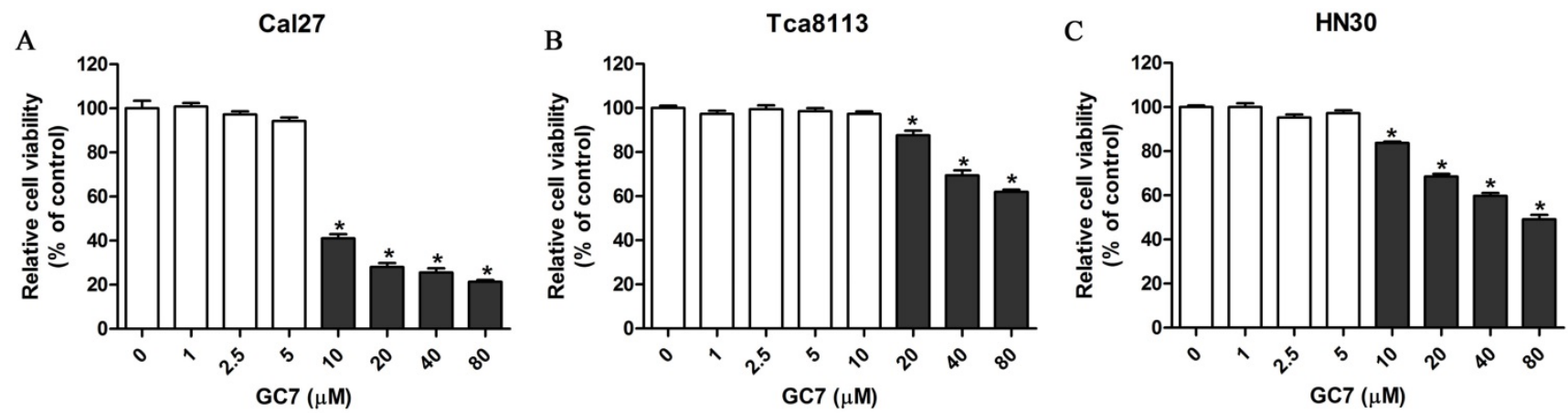

Figure 1. Low doses of N1-guanyl-1,7-diaminoheptane (GC7) had little on cytotoxicity against oral squamous cell carcinoma (OSCC) cells. Cal27 (A), Tca8113 (B), and HN30 (C) cells were treated by indicated doses of GC7 for 48 h. The CCK-8 values were normalized to the negative control group (control), which was treated without GC7. * $\mathrm{p}<0.05$. 
demonstrated that GC7 could prevent eIF5A-2 hypusination efficiently at its low concentrations in several human cancer cell lines. In the present study, 5 $\mu$ M GC7, which exerted little cytotoxic effect against OSCC cells but could suppress the efficiency of eIF5A-2 activity, was used in the following co-treatment with doxorubicin.

\section{GC7 boosted the sensitivity of doxorubicin in OSCC cell lines}

Treated with doxorubicin or doxorubicin combined with GC7 for $48 \mathrm{~h}$, the synergistic cytotoxicity of doxorubicin combined with GC7 in OSCC cells was evaluated using the CCK-8 assay and EdU incorporation assay. Our data revealed that increasing concentrations of doxorubicin inhibited cell viability in all OSCC cell lines (Figure 2). Doxorubicin was more sensitive to Cal27 and HN30 cells than Tca8113 cells. The $\mathrm{IC}_{50}$ values of
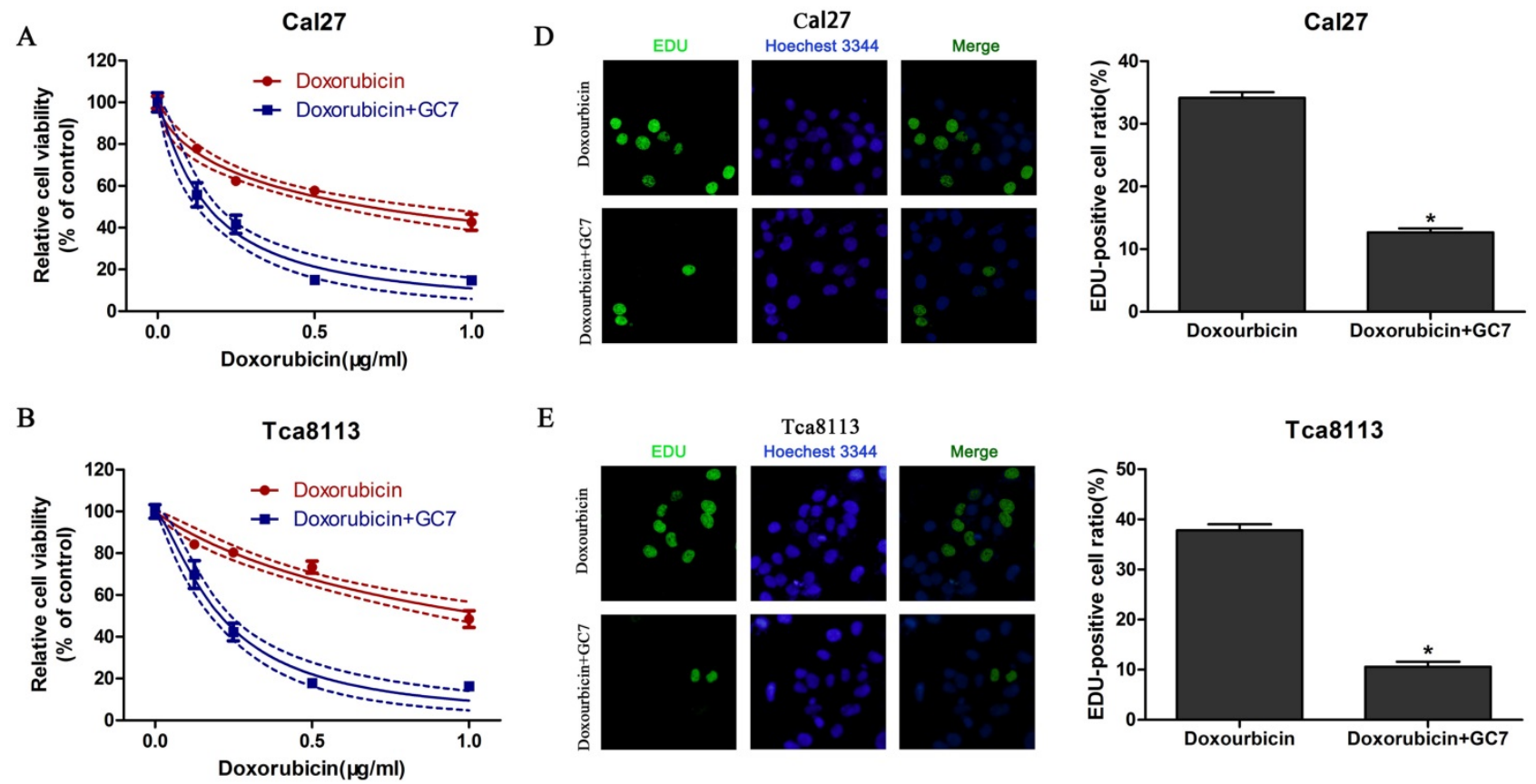

E
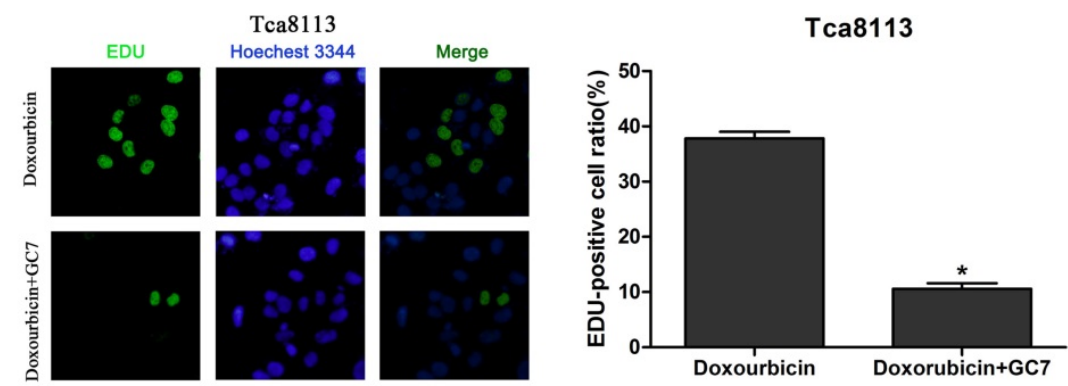

C

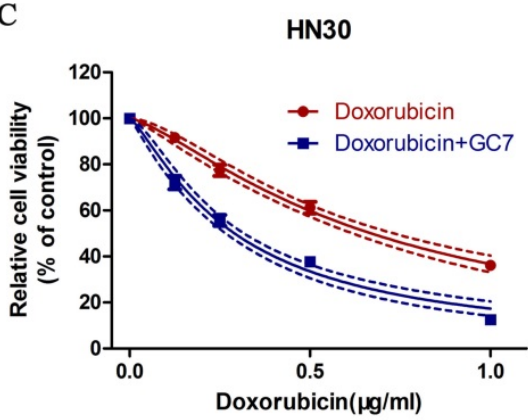

F

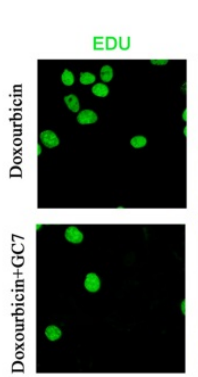

HN30
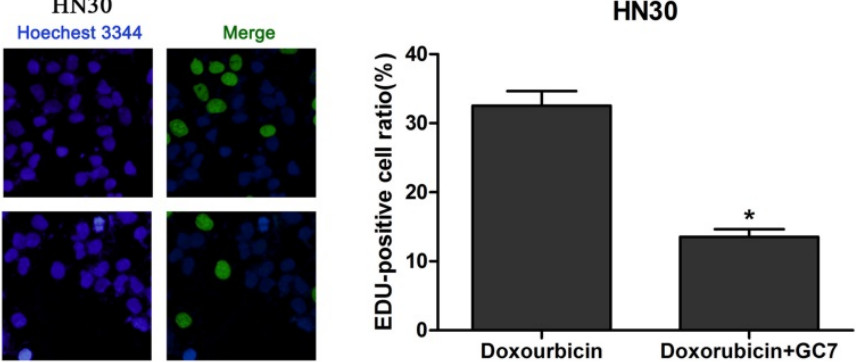

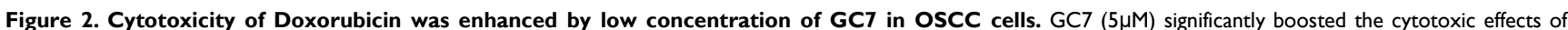
Doxorubicin in Cal27 (A), Tca8113 (B), and HN30 (C) cells. The best fit lines (solid) and 95\% confidence intervals (dashed) are indicated for the treatments in different cells. After $48 \mathrm{~h}$ of intervention by Doxorubicin alone or Doxorubicin+GC7, the staining pattern of 5-ethynyl-2'-deoxyuridine (EdU) and relative EdU-positive ratio were presented, respectively, of Cal27 (D), Tca8113 (E), and HN30 (F) cells. * $\mathrm{p}<0.05$ for Doxorubicin+GC7 vs. Doxorubicin. 
In order to investigate the phenotypes of three OSCC cell lines, expression levels of EMT markers, E-cadherin (epithelial) and Vimentin (mesenchymal), were examined using western blotting and measured the ratio of E-cadherin/Vimentin in all three OSCC cell lines. The ratio of E-cadherin/Vimentin was obviously higher in Cal27 cells which have an epithelial phenotype (Figure 3A, B) than HN30 and Tca8113 cells with a mesenchymal phenotype (Figure $3 \mathrm{~A}, \mathrm{C}, \mathrm{D})$. The results indicated that the capability of GC7 to boost the cytotoxic effect of doxorubicin did not differ from phenotypes of OSCC cells.

\section{GC7 reversed doxorubicin-induced EMT process in OSCC cell lines}

Despite the therapeutic effects of doxorubicin, EMT process is also induced simultaneously. As a result, malignant properties of human cancer cells are enhanced including chemo-resistance and metastasis. To explore whether the EMT process was induced by doxorubicin in OSCC cell lines, we examined EMT molecular markers in OSCC cells during treatment with doxorubicin. Our results revealed that doxorubicin significantly reduced E-cadherin expression and increased Vimentin expression in Cal27 cells with an epithelial phenotype (Figure 3A, B). Remarkably, doxorubicin also induced similar results in HN30 and Tca8113 cells with a mesenchymal phenotype (Figure 3A, C, D). These findings showed that doxorubicin not only induced EMT in epithelial OSCC cells but also further downregulated E-cadherin expression and upregulated Vimentin expression in mesenchymal OSCC cells.

The reverse ratio of E-cadherin/Vimentin is considered to induce the EMT process in cancer cells, which sequentially makes cancer cells more resistant to chemotherapeutic agents. In order to explore whether GC7 can reverse the doxorubicin-induced EMT process, we detected the expression levels of EMT molecular markers in OSCC cells treated by doxorubicin plus GC7. There was a dramatic upregulated expression of E-cadherin and downregulated expression of Vimentin in epithelial Cal27 cells when co-incubation with doxorubicin and GC7, compared to incubated with doxorubicin alone (Figure 3A, B). The E-cadherin/Vimentin ratio in Cal27 cells co-incubated with doxorubicin and GC7 were even higher than untreated control cells. These findings showed that doxorubicin-induced EMT process could be reversed by GC7 in epithelial phenotype OSCC cells. In addition, co-incubation with doxorubicin and GC7 not only increased expression of E-cadherin and decreased expression of Vimentin but also reversed their epithelial/ mesenchymal ratio in mesenchymal HN30 and Tca8113 cells (Figure 3A, C, D). Hence, our data suggested that the combined treatment of GC7 and doxorubicin which lead to the mesenchymalepithelial transition (MET) process might contribute to the enhanced sensitivity of doxorubicin in mesenchymal phenotype OSCC cells.

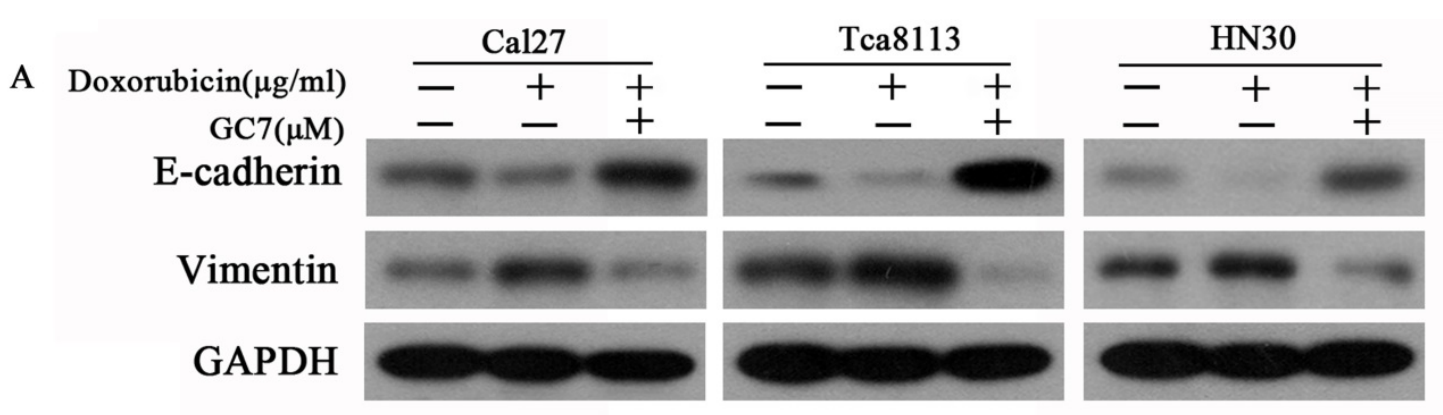

B

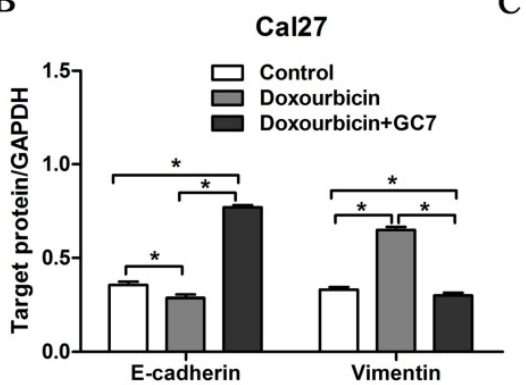

$\mathrm{C}$

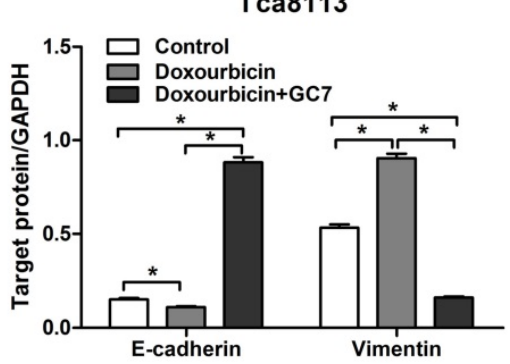

HN30

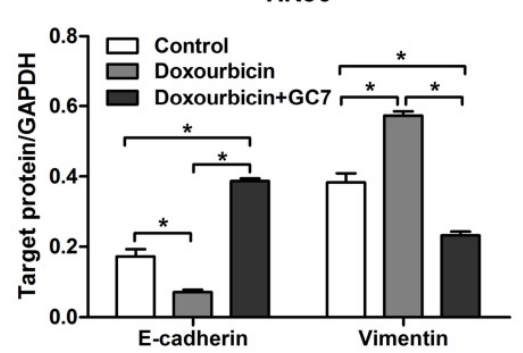

Figure 3. GC7 reversed the alterations of epithelial-mesenchymal transition (EMT) markers expression in Doxorubicin-treated OSCC cells. Expression of E-cadherin and Vimentin, the most important EMT markers, were studied by Western blotting (A) in OSCC Cal27, Tca8113 and HN30 cells treated with negative control, Doxorubicin or Doxorubicin+GC7 for $48 \mathrm{~h}$. Indicated protein expression levels in Cal27 (B), Tca81 13 (C), and HN30 (D) cells were measured by relative band density ratios with GAPDH. $* p<0.05$. 
A

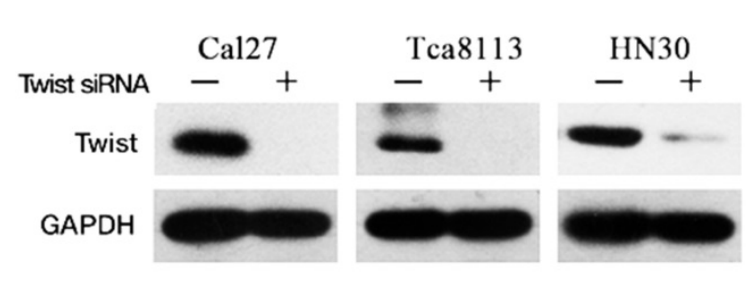
B Cal27

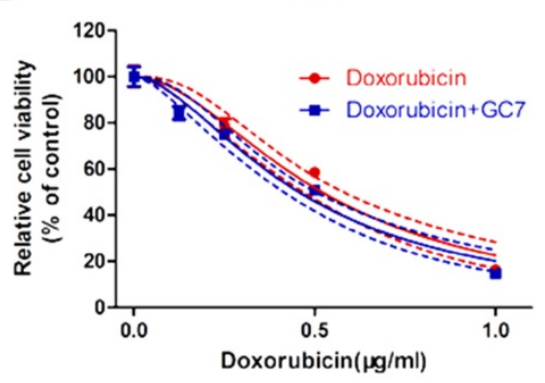

C

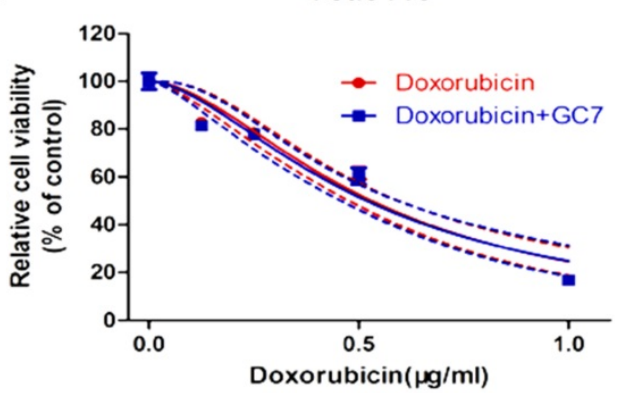

D

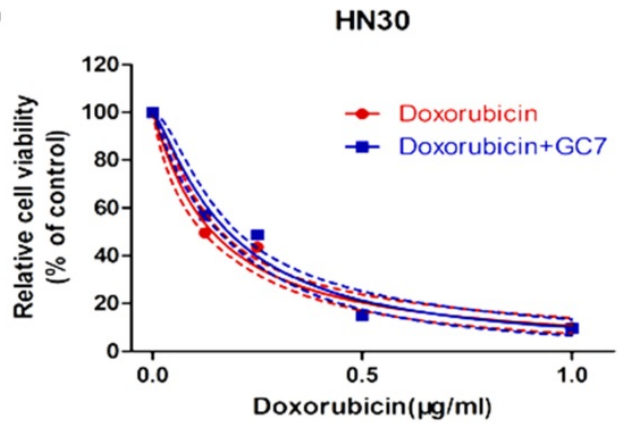

Figure 4. Effects of Twist siRNA on the cytotoxic effect of Doxorubicin or Doxorubicin combined with GC7 in OSCC cells. Western blotting analysis (A) of Twist expression in Twist-siRNA (+) or Negative control-siRNA (-) transfected OSCC cells. Knockdown of Twist by siRNA-transfection reduced the synergistic cytotoxic effect of Doxorubicin and GC7 combination treatment in Cal27 (B), Tca8113 (C), and HN30 (D) cells. The best fit lines (solid) and $95 \%$ confidence intervals (dashed) are indicated for the treatments in different cells. $\mathrm{p}>0.05$ for Doxorubicin $+\mathrm{GC7}$ vs. Doxorubicin.

\section{EMT involved in the resistance of OSCC cells to doxorubicin}

To determine whether EMT involved in the resistance of OSCC cells to doxorubicin, expression of Twist, a transcription factor known to drive EMT and metastasis, was significantly inhibited using siRNA transfection in doxorubicin-treated Cal27, HN30 and Tca8113 cells (Figure 4A). These results suggested that the EMT process induced by doxorubicin might be reversed via Twist silencing in OSCC cells. After treating Twist siRNA transfected OSCC cells with doxorubicin, or doxorubicin plus GC7, we found that Twist siRNA treatment increased the sensitivity of doxorubicin compared to the untreated OSCC cells (supplemental). As expected, when Twist siRNA transfected OSCC cells were co-treated with doxorubicin plus GC7, GC7 had little effect on the doxorubicin chemosensitivity compared to doxorubicin alone (Figure 4B-D, see more in supplemental). Therefore, EMT caused by doxorubicin was responsible for its chemoresistance in OSCC cells and sensitivity of doxorubicin could be enhanced by GC7 mainly though reversing EMT.

\section{Inhibiting expression of elF5A-2 attenuated doxorubicin-caused EMT in OSCC cells}

GC7 is identified to specifically inhibit the function of DHPS, which facilitates the post-translation modifications essential to eIF5A-2 activation. Our previous data have shown that GC7 could enhance the sensitivity of doxorubicin by reversing doxorubicin-induced EMT in OSCC cell lines. To determine whether eIF5A-2 was essential to the EMT process caused by doxorubicin, expression of eIF5A-2 was inhibited using siRNA transfection in OSCC cells (Figure 5A). Treated with doxorubicin alone or doxorubicin combined with GC7 for $48 \mathrm{~h}$, eIF5A-2 siRNA transfected OSCC cells revealed more sensitive to doxorubicin (supplemental). Remarkably, when doxorubicin co-treated with GC7 in eIF5A-2 siRNA transfected OSCC cells, little difference was observed in the doxorubicin chemosensitivity compared to doxorubicin alone (supplemental). Treating eIF5A-2 siRNA transfected Cal27 cells with doxorubicin for $48 \mathrm{~h}$, western blot revealed the increased expression of E-cadherin and decreased expression of vimentin compared to negative control siRNA transfected Cal27 cells, and as a result, the eIF5A-2 siRNA transfected Cal27 cells maintained as epithelial phenotype (Figure 5A, B). Moreover, our results also revealed the reversed expression pattern of E-cadherin and vimentin in eIF5A-2 siRNA transfected HN30 and Tca8113 cells compared to negative control siRNA transfected cells (Figure 5A, C, D), which indicated mesenchymal-epithelial phenotype transition. Our results demonstrated that eIF5A-2 was essential to the EMT process caused by doxorubicin in OSCC cells in vitro. 
A

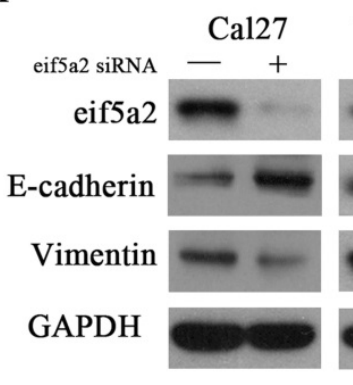

C

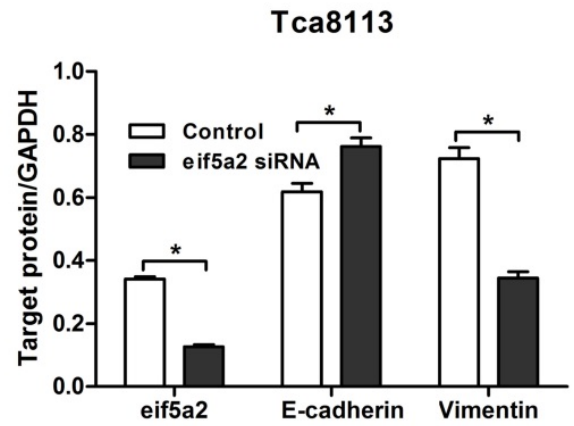

B

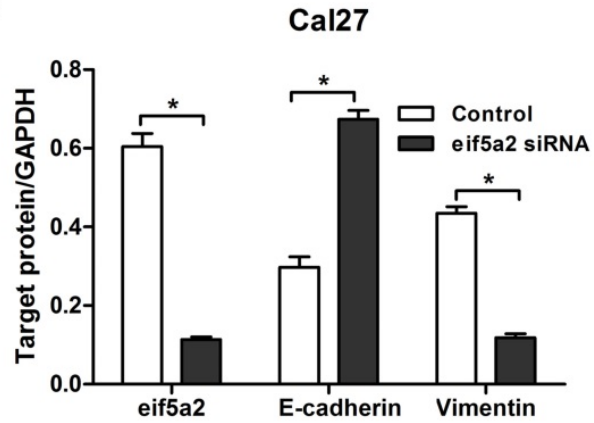

D

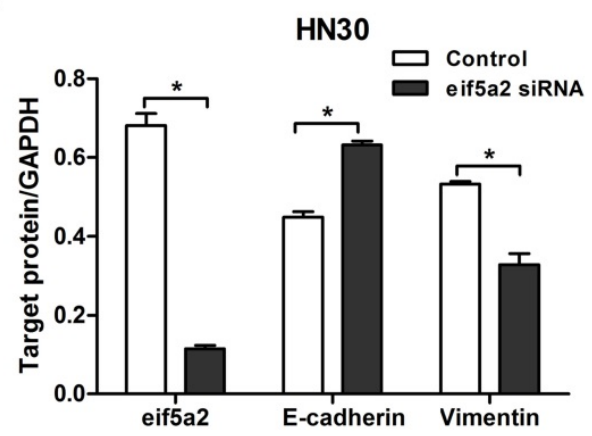

Figure 5. Doxorubicin-induced EMT process was reversed by transfection of elF5A-2 siRNA in OSCC cells. Protein expression of elF5A-2, E-cadherin and Vimentin in elF5A-2-siRNA (+) or Negative control-siRNA (-) transfected OSCC cells treated with Doxorubicin for additional $48 \mathrm{~h}$ were examined by Western blotting analysis (A). Indicated protein expression levels in Cal27 (B), Tca8113 (C), and HN30 (D) cells were measured by relative band density ratios with GAPDH. * $\mathrm{p}<0.05$.

\section{GC7 enhanced the chemotherapeutic effect of doxorubicin in OSCC cells in vivo}

Based on the significantly enhanced cytotoxicity of doxorubicin by GC7 which played an essential role of doxorubicin-induced EMT regulation in OSCC cell lines in vitro, we studied the in vivo chemotherapeutic effect of doxorubicin/GC7 combination in mesenchymal OSCC Tca8113 cells. The tumor volume decrease was not statistically significant during 12 days of GC7 treatment after Tca8113 cells implantation for 2 weeks, while a decreased trend could be detected (Figure 6A, B, D). As shown in Figure 6, doxorubicin alone exhibited a significant reduction of the OSCC growth compared to the control, as revealed by measuring tumor volume during the process of intervention therapy. The tumor weight reduction was further observed significantly at $12^{\text {th }}$ day of doxorubicin treatment when animals were euthanized (Figure 6C). While doxorubicin was co-administrated with GC7, the inhibiting effect on tumor volume and weight were even more significant than doxorubicin alone (Figure 6). Our data strongly suggested that GC7 may boost the chemotherapeutic effect of doxorubicin in OSCC cells in vivo.

\section{Discussion}

As known, most of the cancer mortality was not caused by the impact of the primary tumors but was related to cancer metastasis. Although OSCC is relatively easy to detect at an early stage at the clinic, the high mortality is remained due to the failure to inhibit cancer metastasis or recurrence [26]. As an important part in the comprehensive treatment of OSCC, adjuvant or neoadjuvant chemotherapy is effective and indispensable, especially for the late or advanced stage patients [27]. However, most traditional chemotherapeutic drugs do not show reliable therapeutic effects, and the patients often have relatively high rates of recurrence or metastasis. Thus, the overall 5-year survival rate for OSCC patients, which has not been considerably improved in the last two decades, is remained significantly lower than many other human cancers[26]. To improve the prognosis and increase the 5-year survival rate of OSCC patients, further understanding of the specific molecules, aberrantly expressed during OSCC chemotherapy is highly desirable for more effective comprehensive treatment. Recently, therapeutic interventions based on molecular targeted anti-tumor drugs and traditional chemotherapeutic agents have revealed to boost the chemo-cytotoxic effects and relieved the related severe complications effectively in OSCC patients [3-5, 7, 22]. In the present study, we first reported whether doxorubicin-based combined therapy with GC7, one of the eIF5A-2 activation inhibitors, could boost the chemo-cytotoxic effect of doxorubicin in OSCC cells. We found that GC7 significantly enhanced the chemo-sensitivity of 


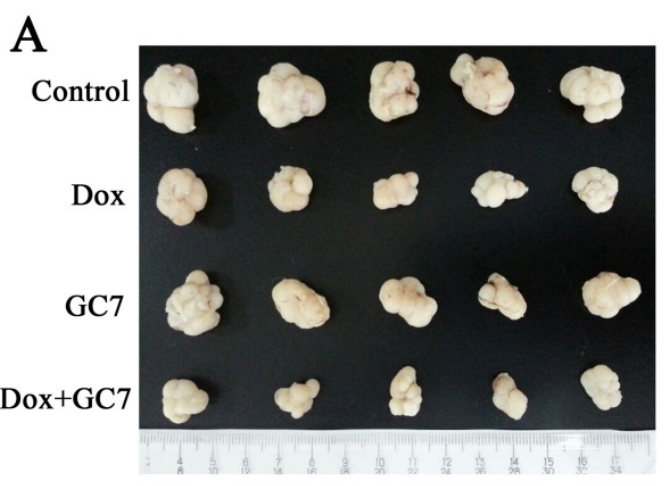

B

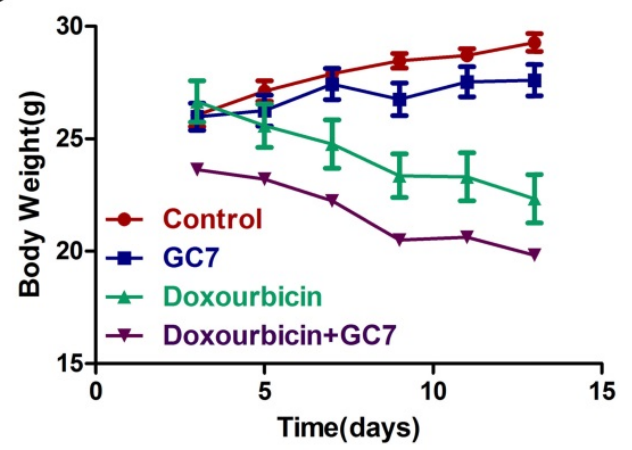

C

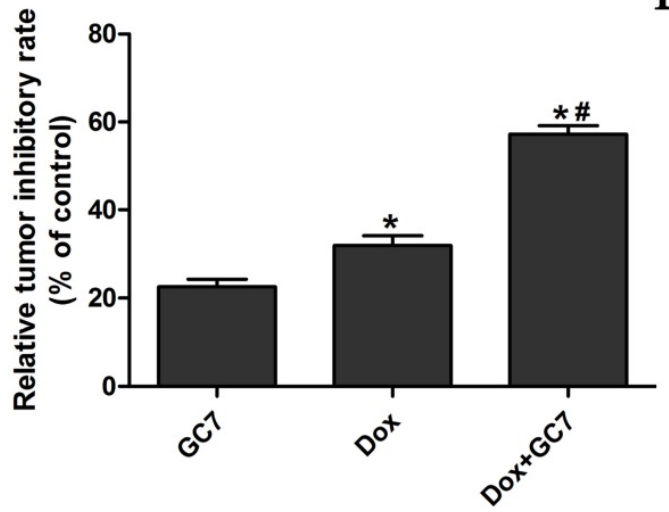

$\mathrm{D}$

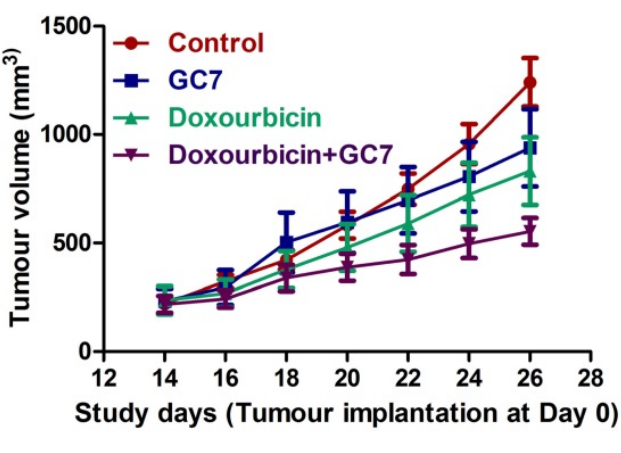

Figure 6. GC7 combined with Doxorubicin revealed potent growth inhibition in mesenchymal phenotype OSCC Tca8113 cells in vivo. Tumor volume (D) and body weight (B) from Tca8113 cells xenografts mice treated with GC7, Doxorubicin, Doxorubicin+GC7 or vehicle-treated negative control groups were evaluated every other day as indicated. After 2 weeks of intervention, tumor specimens were dissected and weighted after euthanization (A). (C) Relative tumor inhibitory rates were standardized to control group. * $\mathrm{p}<0.05$ for Doxorubicin vs. GC7; *\# $\mathrm{p}<0.05$ for Doxorubicin+GC7 vs. Doxorubicin. Dots, mean; bars, SD.

OSCC Tca8113, HN30 and Cal27 cells to doxorubicin in vitro and in vivo, and also investigated the molecular mechanisms mediating the synergistic effects of OSCC cells to doxorubicin.

EMT, the course through which transforms epithelial phenotype cells into mesenchymal phenotype cells, is known to be vital to develop chemo-resistance in many human cancers, including OSCC [8]. In our study, doxorubicin downregulated E-cadherin and upregulated vimentin in Cal27, Tca8113 and HN30 cells, which indicated that OSCC cells underwent the EMT in response to the chemotherapeutic drug. Combined treatment with doxorubicin and GC7, we found that mesenchymal Tca8113 and HN30 cells transformed into epithelial phenotype. In epithelial cells, when co-incubated with doxorubicin and GC7, doxorubicin-induced EMT was reversed in Cal27 cells. Furthermore, GC7 significantly increased Cal27, Tca8113 and HN30 cells sensitivity to doxorubicin. Our findings suggested that doxorubicin-induced EMT could be inhibited and even reversed by GC7 in both mesenchymal and epithelial phenotype OSCC cells.

To further investigate the molecular mechanisms of GC7 in the doxorubicin-induced EMT, eIF5A-2 siRNA was transfected into the three OSCC cell lines.
As expected, knockdown of eIF5A-2 made OSCC cells more sensitive to doxorubicin. However, the synergistic effect of combined treatment of doxorubicin and GC7 was abolished after transfection of the eIF5A-2 siRNA. Moreover, the eIF5A-2 siRNA transfection showed upregulation of E-cadherin expression and downregulation of Vimentin expression in doxorubicin pre-treated OSCC cells. During the process of EMT, expression switching of cadherin from E-cadherin to $\mathrm{N}$-cadherin drives cell invasion and migration, which relates to poor prognosis [28, 29]. It has been demonstrated that Twist (a transcription factor known to drive EMT and metastasis) is involved with the induction of $\mathrm{N}$-cadherin in addition to its suppressive effect on E-cadherin expression [9, 10]. Interestingly, Twist siRNA transfected OSCC cells showed little effect in the doxorubicin sensitivity combined with GC7 compared to doxorubicin alone, which further confirmed that GC7 enhanced OSCC to doxorubicin sensitivity through EMT molecular pathway regulation.

Currently, novel therapeutic interventions, based on targeting specific genes, abnormally regulated in OSCC carcinogenesis, have been studied in several pre-clinical trials with promising results 
[30-32]. Our final aim for the future is to substitute traditional chemotherapies which are considered nonspecific and cause unwanted severe complications in patients. DHPS is highly related to cancer metastasis [33] and GC7, an inhibitor of DHPS activity, has been studied in several human cancers $[34,35]$. Several studies have reported that GC7 could induce tumorigenicity reduction, cancer cell migration suppression and the EMT reversion in non-small cell lung cancer [14], hepatocellular carcinoma [21], pancreatic cancer [36] and melanoma [37]. It is also known that EMT can be induced by doxorubicin in some human cancers and we have first demonstrated it in OSCC; meanwhile, molecular mechanisms which might be responsible for EMT process induced by doxorubicin in OSCC has also been studied. Our results have demonstrated the involvement of eIF5A-2 in the EMT signaling pathway regulation, which could contribute to the chemo-resistance of OSCC cells to doxorubicin. Interestingly, different with bladder cancer and hepatocellular carcinoma, our data proved that combination therapy with GC7 was to not only increase the efficacy of doxorubicin in epithelial phenotype OSCC cells but also to eradicate the more aggressive mesenchymal phenotype OSCC cells. Our results have also strongly suggested that the dose of doxorubicin could be significantly reduced via GC7 combination treatment, which contributed to decrease the influence of the cytotoxic side-effects resulted from high doses of doxorubicin in OSCC treatment. Our study presents the promising prospect of OSCC therapeutic interventions with the combination of doxorubicin and agents targeting the eIF5A-2 signaling pathway. However, further studies are required to investigate and develop our objectives.

\section{Conclusions}

We demonstrate that the chemotherapeutic effect of doxorubicin could be significantly boosted by combination treatment with GC7 via eIF5A-2 activity inhibition and EMT process reversion in OSCC cells with both epithelial and mesenchymal phenotype. Thus, doxorubicin-based therapeutic interventions combined with GC7 could be more effective for OSCC patients. Undoubtedly, this study will provide a novel strategy to develop the new biomarkers for targeted therapeutics for patients suffered from OSCC in the clinic.

\section{Supplementary Material}

Supplementary figure and tables. http://www.jcancer.org/v09p3479s1.pdf

\section{Acknowledgments}

This research was supported by the National Natural Science Foundation of P.R. China (Grant 81302353).

\section{Ethics}

All applicable international, national, and/or institutional guidelines for the care and use of animals were followed. All animal studies were proved by the ethics committee of Sir Run Run Shaw Hospital, School of Medicine, Zhejiang University and performed in compliance with the Guidelines for the Care and Use of Laboratory Animals of Zhejiang University, P.R. China.

\section{Competing Interests}

The authors have declared that no competing interest exists.

\section{References}

1. Siegel RL, Miller KD, Jemal A. Cancer Statistics, 2016. Ca-Cancer J Clin. 2016; 66: 7-30

2. Miller KD, Siegel RL, Lin CC, Mariotto AB, Kramer JL, Rowland JH, et al. Cancer treatment and survivorship statistics, 2016. Ca-Cancer J Clin. 2016; 66: 271-89.

3. Saddoughi SA, Garrett-Mayer E, Chaudhary U, O'Brien PE, Afrin LB, Day TA, et al. Results of a Phase II Trial of Gemcitabine Plus Doxorubicin in Patients with Recurrent Head and Neck Cancers: Serum C-18-Ceramide as a Novel Biomarker for Monitoring Response. Clin Cancer Res. 2011; 17: 6097-105.

4. Argiris A, Ghebremichael M, Burtness B, Axelrod RS, Deconti RC, Forastiere AA. A Phase 2 Trial of Bortezomib Followed by the Addition of Doxorubicin at Progression in Patients With Recurrent or Metastatic Adenoid Cystic Carcinoma of the Head and Neck A Trial of the Eastern Cooperative Oncology Group (E1303). Cancer. 2011; 117: 3374-82.

5. Fountzilas $G$ Papakostas $P$ Dafni $U$ Makatsoris $T$, Karina $M$ Kalogera-Fountzila A, et al. Paclitaxel and gemcitabine vs. paclitaxel and pegylated liposomal doxorubicin in advanced non-nasopharyngeal head and neck cancer. An efficacy and cost analysis randomized study conducted by the Hellenic Cooperative Oncology Group. Ann Oncol. 2006; 17: 1560-7.

6. Krebs LJ, Wang XP, Nagy A, Schally AV, Prasad PN, Liebow C. A conjugate of doxorubicin and an analog of Luteinizing Hormone-Releasing Hormone shows increased efficacy against oral and laryngeal cancers. Oral Oncol. 2002; 38: 657-63.

7. Caponigro F, Avallone A, Rivellini F, Budillon A, Di Gennaro E, Ionna F, et al. Phase I study of Caelyx (doxorubicin HCL, pegylated liposomal) in recurrent head and neck cancer (HN). Clin Cancer Res. 1999; 5: 3819s-s.

8. Krisanaprakornkit S, Iamaroon A. Epithelial-mesenchymal transition in oral squamous cell carcinoma. ISRN Oncol. 2012; 2012: 681469.

9. Yang J, Mani SA, Weinberg RA. Exploring a new twist on tumor metastasis. Cancer Res. 2006; 66: 4549-52.

10. Yang J, Mani SA, Donaher JL, Ramaswamy S, Itzykson RA, Come C, et al. Twist, a master regulator of morphogenesis, plays an essential role in tumor metastasis. Cell. 2004; 117: 927-39.

11. Liu Y, Du FY, Chen W, Yao MY, Lv KZ, Fu PF. EIF5A2 is a novel chemoresistance gene in breast cancer. Breast Cancer. 2015; 22: 602-7.

12. Bao Y, Lu YL, Wang X, Feng WM, Sun XR, Guo HH, et al. Eukaryotic translation initiation factor 5A2 (eIF5A2) regulates chemoresistance in colorectal cancer through epithelial-mesenchymal transition. Cancer Cell International. 2015; 15.

13. Yang J, Yu H, Shen M, Wei W, Xia L, Zhao P. N1-guanyl-1,7-diaminoheptane sensitizes bladder cancer cells to doxorubicin by preventing epithelial-mesenchymal transition through inhibition of eukaryotic translation initiation factor 5A2 activation. Cancer Sci. 2014; 105: 219-27.

14. Xu G, Yu H, Shi X, Sun L, Zhou Q, Zheng D, et al. Cisplatin sensitivity is enhanced in non-small cell lung cancer cells by regulating epithelial-mesenchymal transition through inhibition of eukaryotic translation initiation factor 5A2. Bmc Pulm Med. 2014; 14: 174

15. Mathews MB, Hershey JWB. The translation factor eIF5A and human cancer. Bba-Gene Regul Mech. 2015; 1849: 836-44.

16. Wang FW, Guan XY, Xie D. Roles of Eukaryotic Initiation Factor 5A2 in Human Cancer. Int J Biol Sci. 2013; 9: 1013-20. 
17. Clement PMJ, Henderson CA, Jenkins ZA, Smit-McBride Z, Wolff EC, Hershey JWB, et al. Identification and characterization of eukaryotic initiation factor 5A-2. Eur J Biochem. 2003; 270: 4254-63.

18. Xu GD, Shao GF, Pan QL, Sun LB, Zheng DW, Li MH, et al. MicroRNA-9 regulates non-small cell lung cancer cell invasion and migration by targeting eukaryotic translation initiation factor 5A2. American Journal of Translational Research. 2017; 9: 478-88.

19. Cao TT, Lin SH, Fu L, Tang Z, Che CM, Zhang LY, et al. Eukaryotic translation initiation factor $5 \mathrm{~A} 2$ promotes metabolic reprogramming in hepatocellular carcinoma cells. Carcinogenesis. 2017; 38: 94-104.

20. Yang $\mathrm{H}, \mathrm{Li}$ XD, Zhou Y, Ban XJ, Zeng TT, Li L, et al. Stemness and chemotherapeutic drug resistance induced by EIF5A2 overexpression in esophageal squamous cell carcinoma. Oncotarget. 2015; 6: 26079-89.

21. Tang DJ, Dong SS, Ma NF, Xie D, Chen LL, Fu L, et al. Overexpression of Eukaryotic Initiation Factor 5A2 Enhances Cell Motility and Promotes Tumor Metastasis in Hepatocellular Carcinoma. Hepatology. 2010; 51: 1255-63.

22. Wang $X$, Jiang $R$, Cui $E H$, Feng $W M$, Guo $H H, G u ~ D H$, et al. N1-guanyl-1,7-diaminoheptane enhances the chemosensitivity of NSCLC cells to cetuximab through inhibition of eukaryotic translation initiation factor 5A2 activation. Eur Rev Med Pharmaco. 2016; 20: 1244-50.

23. Lee SK, Lee J, Lee SI, Bae WJ, Lee YM, Park JS, et al. $\mathrm{N}(1)$-guanyl-1,7,-diamineoheptane, an inhibitor of deoxyhypusine synthase, suppresses differentiation and induces apoptosis via mitochondrial and AMPK pathways in immortalized and malignant human oral keratinocytes. J Oral Pathol Med. 2009; 38: 792-800.

24. Shi XP, Yin KC, Ahern J, Davis LJ, Stern AM, Waxman L. Effects of N1-guanyl-1,7-diaminoheptane, an inhibitor of deoxyhypusine synthase, on the growth of tumorigenic cell lines in culture. Biochim Biophys Acta. 1996; 1310: 119-26.

25. Fang L, Wang H, Zhou L, Yu D. FOXO3a reactivation mediates the synergistic cytotoxic effects of rapamycin and cisplatin in oral squamous cell carcinoma cells. Toxicol Appl Pharmacol. 2011; 251: 8-15.

26. Scully C, Bagan J. Oral squamous cell carcinoma overview. Oral Oncol. 2009; 45: 301-8

27. Huang SH, O'Sullivan B. Oral cancer: Current role of radiotherapy and chemotherapy. Med Oral Patol Oral. 2013; 18: E233-E40.

28. Wheelock MJ, Shintani Y, Maeda M, Fukumoto Y, Johnson KR. Cadherin switching. J Cell Sci. 2008; 121: 727-35.

29. Lehembre F, Yilmaz M, Wicki A, Schomber T, Strittmatter K, Ziegler D, et al. NCAM-induced focal adhesion assembly: a functional switch upon loss of E-cadherin. EMBO J. 2008; 27: 2603-15.

30. Sasahira T, Kirita T, Kuniyasu H. Update of molecular pathobiology in oral cancer: a review. Int J Clin Oncol. 2014; 19: 431-6.

31. Hamakawa H, Nakashiro K, Sumida T, Shintani S, Myers JN, Takes RP, et al. Basic evidence of molecular targeted therapy for oral cancer and salivary gland cancer. Head Neck. 2008; 30: 800-9.

32. Choi S, Myers JN. Molecular pathogenesis of oral squamous cell carcinoma: implications for therapy. J Dent Res. 2008; 87: 14-32.

33. Ramaswamy S, Ross KN, Lander ES, Golub TR. A molecular signature of metastasis in primary solid tumors. Nat Genet. 2003; 33: 49-54

34. Bandino A, Geerts D, Koster J, Bachmann AS. Deoxyhypusine synthase (DHPS) inhibitor GC7 induces p21/Rb-mediated inhibition of tumor cell growth and DHPS expression correlates with poor prognosis in neuroblastoma patients. Cell Oncol (Dordr). 2014; 37: 387-98.

35. Lou B, Fan J, Wang KY, Chen W, Zhou XQ Zhang J, et al N1-guanyl-1,7-diaminoheptane (GC7) enhances the therapeutic efficacy of doxorubicin by inhibiting activation of eukaryotic translation initiation factor 5A2 (eIF5A2) and preventing the epithelial-mesenchymal transition in hepatocellular carcinoma cells. Experimental Cell Research. 2013; 319: 2708-17.

36. Fujimura K, Wright T, Strnadel J, Kaushal S, Metildi C, Lowy AM, et al. A hypusine-eIF5A-PEAK1 switch regulates the pathogenesis of pancreatic cancer. Cancer Res. 2014; 74: 6671-81.

37. Jasiulionis MG, Luchessi AD, Moreira AG, Souza PP, Suenaga AP, Correa M, et al. Inhibition of eukaryotic translation initiation factor 5A (eIF5A) hypusination impairs melanoma growth. Cell Biochem Funct. 2007; 25: 109-14. 